\title{
NIR assignment of isopsoralen by 2D-COS technology and model application in Yunkang Oral Liquid
}

\author{
Yanling Pei, Zhisheng $\mathrm{Wu}^{*, \S}$, Xinyuan Shi, Xiaoning Pan, \\ Yanfang Peng and Yanjiang Qiao ${ }^{\dagger, \S}$ \\ Beijing University of Chinese Medicine \\ Beijing 100102, P. R. China \\ Beijing Key Laboratory for Basic and \\ Development Research on Chinese \\ Medicine, Beijing 100102, P. R. China \\ Key Laboratory of TCM-Information Engineer \\ of State Administration of TCM \\ Beijing 100102 P. R. China \\ *wzs@bucm.edu.cn \\ †yjqiao@263.net
}

Received 9 October 2014

Accepted 25 November 2014

Published 31 December 2014

\begin{abstract}
Near infrared (NIR) assignment of Isopsoralen was performed using deuterated chloroform solvent and two-dimensional correlation spectroscopy (2D-COS) technology. Yunkang Oral Liquid was applied to study Isopsoralen, the characteristic bands by spectral assignment as well as the bands by interval partial least squares (iPLS) and synergy interval partial least squares (siPLS) were used to establish partial least squares (PLS) model. The coefficient of determination in calibration $\left(R_{\text {cal }}^{2}\right)$ were $0.9987,0.9970$ and 0.9982 . The coefficient of determination in cross validation $\left(R_{\text {val }}^{2}\right)$ were $0.9985,0.9921$ and 0.9982 . The coefficient of determination in prediction $\left(R_{\text {pre }}^{2}\right)$ were $0.9987,0.9955$ and 0.9988 . The root mean square error of calibration (RMSEC) were $0.27,0.40$ and $0.31 \mathrm{ppm}$. The root mean square error of cross validation (RMSECV) were 0.30 , 0.67 and $0.32 \mathrm{ppm}$. The root mean square error of prediction (RMSEP) were $0.23,0.43$ and $0.22 \mathrm{ppm}$. The residual predictive deviation (RPD) were $31.00,16.58$ and 32.41 . It turned out that the characteristic bands by spectral assignment had the same results with the chemometrics methods in PLS model. It provided guidance for NIR spectral assignment of chemical compositions in Chinese Materia Medica (CMM).
\end{abstract}

Keywords: Near infrared spectroscopy; two-dimensional correlation spectroscopy; Isopsoralen; Yunkang Oral Liquid; spectral assignment.

§Corresponding authors.

This is an Open Access article published by World Scientific Publishing Company. It is distributed under the terms of the Creative Commons Attribution 3.0 (CC-BY) License. Further distribution of this work is permitted, provided the original work is properly cited. 


\section{Introduction}

Critical quality attribute (CQA) assessment has been highly regarded by International Conference on Harmonization of Technical Requirements for Registration of Pharmaceuticals for Human Use (ICH) and Food and Drug Administration (FDA) in pharmaceutical manufacture process. Near infrared (NIR) is an excellent and rapid technique for evaluating the CQA because of its fast, nondestructive and real-time advantages. The NIR wavelength is from 780 to $2500 \mathrm{~nm}$. In this area, the main vibrations of functional groups including a hydrogen atom are observed, such as $-\mathrm{CH},-\mathrm{NH},-$ $\mathrm{OH}$ and $-\mathrm{SH}$ bonds. ${ }^{1}$ For the past few years, NIR has become a powerful analytical technique in quantitative and qualitative analysis. ${ }^{2}$ It provided useful functions across a range of application areas, such as pharmacy, chemical industry, food and petroleum field..$^{3,4}$

However, NIR has overlapping and broad absorption bands due to the overtones and combination tones. It is difficult to identify the characteristic peaks in NIR wavelength. There are few researches on NIR spectral assignment. Meanwhile, there is also no report on the NIR assignment of Chinese Materia Medica (CMM). Spectral assignment is significant with regard to accurate quantitative and qualitative analysis in various fields. Thus, NIR spectral assignment is very important in its application. The relationship between spectra and molecular structure enables NIR spectral assignment to achieve accurate quantitative and qualitative analysis.

Two-dimensional correlation spectroscopy (2DCOS) is a powerful and versatile technique to assign the NIR spectra. It was firstly proposed in the field of nuclear magnetic resonance. Then generalized 2D-COS technology expanded a variety of applications, which was proposed by Noda et al. ${ }^{5,6}$ It mainly focused on the external disturbances about spectral variation. It could be applied to find the characteristic bands in complicated spectra. The external disturbances included temperature, pressure and concentration. ${ }^{7,8}$

Liu illuminated the NIR band assignments of methanol and ethanol by generalized 2D-COS. ${ }^{9}$ Genkawa et al. demonstrated the IR/NIR absorption bands of water and liquid oleic acid by 2D-COS technology, in which the temperature was used as external disturbance. ${ }^{10}$ According to the structure and spectra, 2D-COS technology was applied to assign the variation of band position shift by Shinzawa et al. It revealed a phenomenon that the cellulose crystalline phase disorganized in accordance with the grinding. ${ }^{11}$ Other researches have also made significant trials on band assignments of NIR using 2D-COS technology in the references cited. ${ }^{12}$

With a history of 2000 to 3000 years, CMM has formed a unique and complex system to diagnose and cure illness. NIR is a rapid technique for CQA assessment in CMM to guarantee the safety, effectiveness, stability and uniformity. However, when NIR technology has been used in CMM, overlapping absorption, wide peaks and low absorption would appear to limit the application.

To obtain a robust model, variable selection methods were chosen to filter variables, such as interval partial least squares (iPLS), moving window partial least squares (mwPLS), backward interval partial least squares (biPLS) and synergy interval partial least squares (siPLS). Wu reported that iPLS was regarded as the variable selection method for chlorogenic acid in the extraction process of Lonicera japonica. The result demonstrated that NIR allowed the detection of a minor analyte (50 ppm) in CMM. ${ }^{13}$ Wang et al. illuminated that the best spectral band was selected to detect alcohol concentration using siPLS analysis. It showed that the model had good predictive ability and reliability. ${ }^{14}$ Jiang et al. demonstrated that siPLS was used to predict process variables in solid-state fermentation of wheat straw. The results showed that optimal model was achieved by siPLS with selected spectral subintervals. ${ }^{15}$ Chen et al. reported that NIR could determine total polyphenols content in green tealeaves. It showed that siPLS and iPLS were used to establish NIR model, and the siPLS method achieved good predictive ability. ${ }^{16}$

However, when variable selection methods of Chemometrics were used to establish NIR model, the same substance often has different bands due to different methods. ${ }^{17,18}$ In this paper, the expectation about NIR research focused on model interpretation using NIR spectral assignment coupled with the relationship between spectra and molecular structure. Yunkang Oral Liquid is a Chinese herbal preparation, including Codonopsis pilosula Nannf., Poria cocos Wolf., Psoralea corylifolia L., etc. ${ }^{19}$ Yunkang Oral Liquid has the obvious therapeutic effects on enlivening the spleen, stabilizing 
the kidney, nourishing blood and calming abortion. It is used for treating the threatened abortion and habitual abortion. ${ }^{20}$

In this study, 2D-COS technology was introduced and concentration was selected as the external disturbance. NIR spectral assignment of Isopsoralen was performed with deuterated chloroform and the quantitative NIR model about CQA (Isopsoralen) of Yunkang Oral Liquid as the example. The characteristic bands from chemometrics and spectral assignment from 2D-NIR were used to establish quantitative model in Yunkang Oral Liquid. In chemometrics application, siPLS and iPLS methods were used to select the variable of Isopsoralen in Yunkang Oral Liquid. Finally, model interpretation in Yunkang Oral Liquid was demonstrated using NIR spectral assignment coupled with the relationship between spectra and molecular structure.

\section{Materials and Methods}

\subsection{Materials}

Yunkang Oral Liquid was purchased from the market, and deposited in the key laboratory of Traditional Chinese Medicine-information Engineering of State Administration of Traditional Chinese Medicine (No. 140528). Isopsoralen reference standard (lot number: 110738-20131) was supplied by the Chinese Food and Drug Inspection Institute (Beijing, China). Deuterated chloroform was purchased from Cambridge Isotope Laboratories, Inc. (Massachusetts, USA). HPLC grade methanol was purchased from Thermo Fisher Scientific Inc. (Massachusetts, USA). Deionized water was purchased from Hangzhou Wahaha Co., Ltd (Zhejiang, China).

\subsection{Preparation of samples}

For NIR assignment of Isopsoralen, the $50.08 \mathrm{mg}$ of Isopsoralen was weighed accurately into a $10 \mathrm{~mL}$ volumetric flask, and diluted with deuterated chloroform. The solution was obtained including $5.008 \mathrm{mg}$ of Isopsoralen in each of milliliter. Series of percentages about the solution were transferred to $2 \mathrm{~mL}$ volumetric flask separately, and further diluted with deuterated chloroform for 2D-COS analysis, which the concentration level ranged from 1.00 to $5.00 \mathrm{mg} \cdot \mathrm{mL}^{-1} .^{21}$
While applying the NIR assignment of Isopsoralen, 50 samples of Yunkang Oral Liquid diluted with water were obtained containing series of Isopsoralen concentration levels, ranging from $2 \%$ to $100 \%(\mathrm{v} / \mathrm{v})$. In order to obtain a robust NIR model, 50 samples were selected, 33 as calibration sets (validation sets, leave-one cross validation) and 17 as prediction sets according to Kennard-Stone (KS) algorithm.

\subsection{NIR equipment and measurement}

The NIR spectra were collected by XDS analyzer and VISION software (Metrohm NIR System, Florida, USA). The wavelength range of spectra was from 780 to $2500 \mathrm{~nm}$. Each spectrum was an average of 32 scans and 3 times with a wavelength increment of $0.5 \mathrm{~nm}$. Data measurement was performed by VISION software (Vision 3.5, Metrohm NIR System, Florida, USA).

\subsection{HPLC methods}

Chromatographic analysis was carried out on Agilent HPLC apparatus, which was comprised of Agilent 1100 system, an auto-sampler, a column temperature controller and a diode-array detector (DAD) (Agilent Technologies, California, USA). Samples were primarily separated on an Agilent ZORBAX SB C18 column $(5 \mu \mathrm{m}, 4.6 \times 250 \mathrm{~mm}$, Agilent Columns, California, USA) at $25^{\circ} \mathrm{C}$ using the mobile phase of methanol and water $(50: 50, \mathrm{v} / \mathrm{v})$. The detection wavelength was set at $264 \mathrm{~nm} .^{22}$

\subsection{The method of $2 D-C O S$}

2D-COS is an excellent method for NIR spectral assignment. It is performed by synchronous spectrum and asynchronous spectrum. The overlapping bands could be distinguished by the synchronous spectrum. The variable order of various bands could be elucidated by the asynchronous spectrum. The auto-peaks in synchronous spectrum represent the characteristic bands of pharmaceutical ingredients. In addition, the enhancement and debase of crosspeaks reflect functional groups. ${ }^{23,24}$

In our previous work, the number of samples and the intervals of concentration range were illuminated in 2D-COS analysis of NIR assignment. ${ }^{21}$ In this study, Isopsoralen was chosen to obtain the NIR assignment according to the positions of the auto-peaks by $2 \mathrm{D}-\mathrm{COS}$ technology. 


\subsection{Software and data analysis}

The Unscrambler 9.6 software package (CAMO Software AS, Oslo, Norway) was applied to analyze the data. 2D-COS and home-made routines program in MATLAB code (MATLAB v7.0, The MathWorks, MA) was also used. The toolbox of iPLS and siPLS used to select the informative variables were downloaded from http://www.models.kvl.dk/. In addition, the coefficient of determination in calibration $\left(R_{\text {cal }}^{2}\right)$, the coefficient of determination in cross validation $\left(R_{\text {val }}^{2}\right)$, the coefficient of determination in prediction $\left(R_{\mathrm{pre}}^{2}\right)$, the root mean square error of calibration (RMSEC), the root mean square error of cross validation (RMSECV), the root mean square error of prediction (RMSEP) and the residual predictive deviation (RPD) were applied to evaluate the PLS model.

\section{Results and Discussion}

\subsection{Quantitative analysis of Isopsoralen by HPLC method}

High performance liquid chromatography (HPLC) method was applied to determine Isopsoralen in Yunkang Oral Liquid. The HPLC chromatograms of reference and sample are shown in Fig. 1. The retention time of Isopsoralen in sample solution was the same as the reference solution. The calibration curve of the HPLC method was drawn before sample analysis. The calibration curve exhibited good linearity $\left(Y=7 \times 10^{6} X-6.9995, R^{2}=1.0000\right)$ within the content ranging from 0.054 to $0.189 \mu \mathrm{g}$. According to the calibration curve of Isopsoralen, each accurate concentration of Yunkang Oral

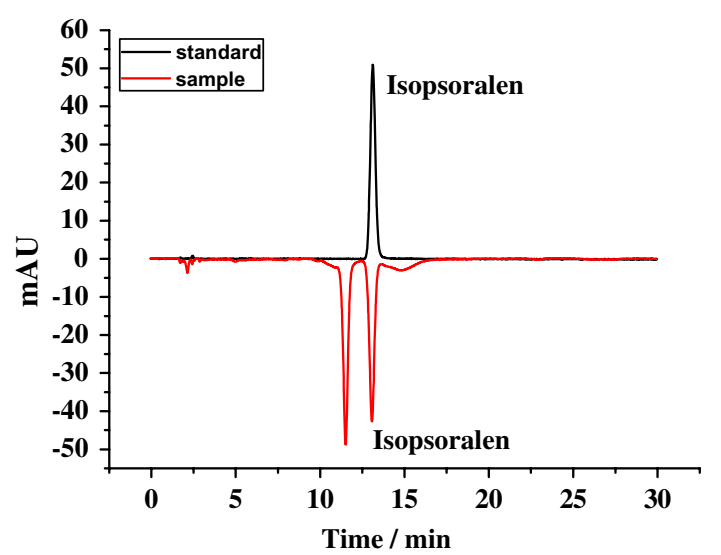

Fig. 1. Chromatograms of reference and Yunkang Oral Liquid.
Liquid was determined, in which the content of Isopsoralen ranged from 0.488 to $24.4 \mu \mathrm{g}$. The mean content was $11.97 \mu \mathrm{g}$.

\subsection{Selection of deuterated chloroform and comparison of different spectral pretreatment methods}

The raw spectra of Yunkang Oral Liquid are shown in Fig. 2(a). As we can see, large fluctuations appeared in the region of combinations and first overtones, which are caused by the noise and the strong absorption of water. Meanwhile, spectra of Isopsoralen were obtained using deuterated chloroform solvent as shown in Fig. 2(b). Due to the deuterium atom and simple structure, the NIR absorption of Isopsoralen in deuterated chloroform

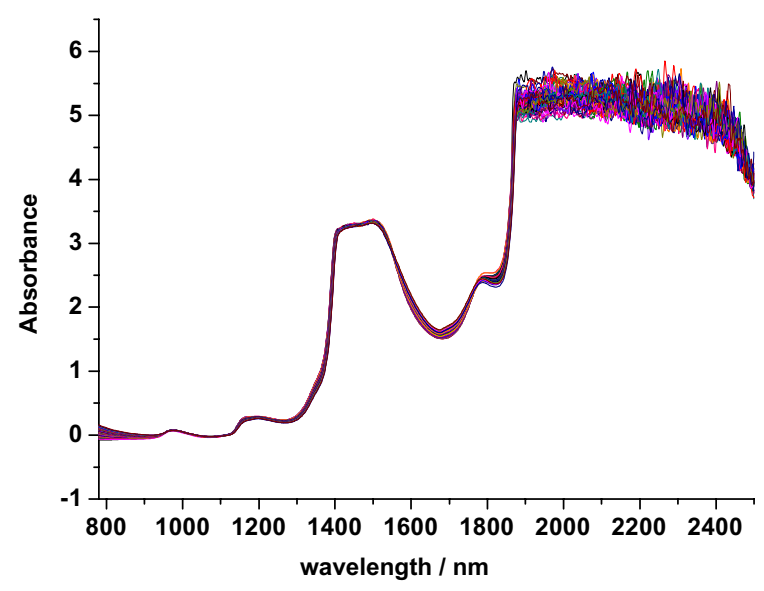

(a)

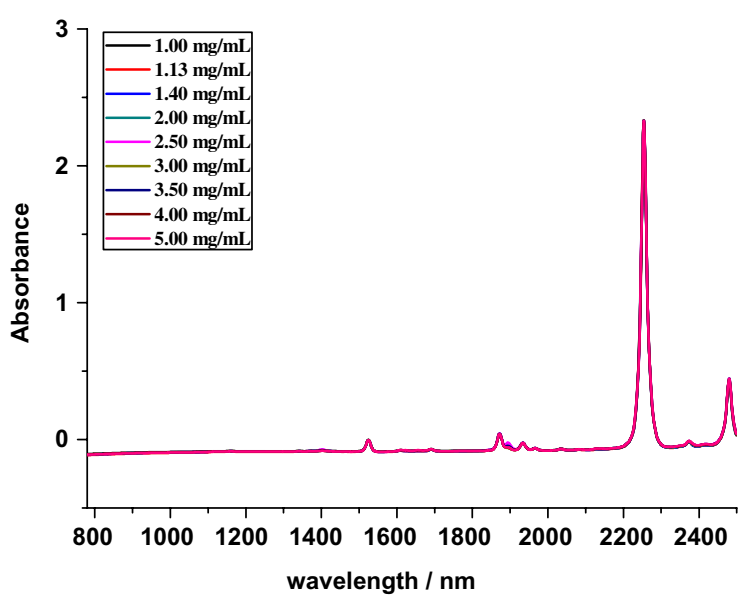

(b)

Fig. 2. The raw spectra of Yunkang Oral Liquid (a) and Isopsoralen dilute in deuterated chloroform (b). 


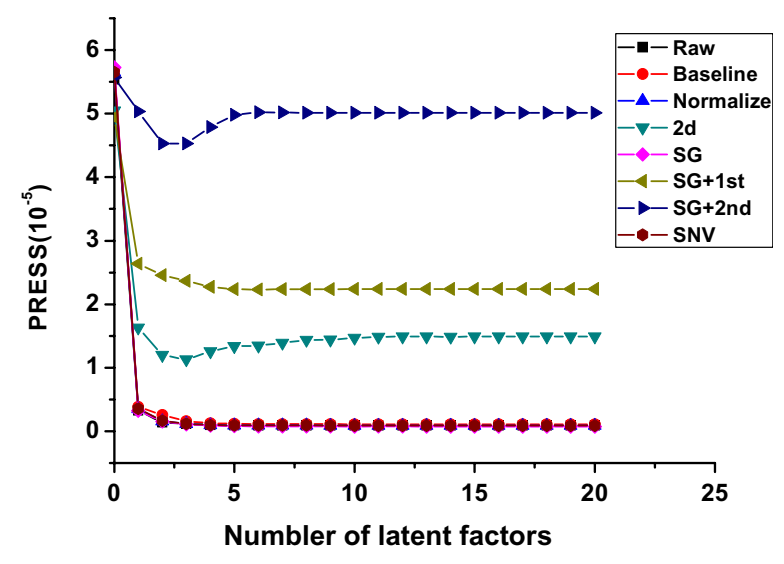

Fig. 3. PRESS plot of NIR by different pretreatments.

was one of the simplest systems, because NIR absorption was mainly from hydrogen groups. Our results proposed that deuterated chloroform was a good solvent for NIR spectral assignment of Isopsoralen.

In addition, spectral pretreatment is very important before establishing a robust model. Different pretreatment methods were used to eliminate the effects of noise and baseline drift and obtain an accurate model. The model performance was excellent according to the following strategies: the coefficient of determination $R^{2}$ was approximately equal to 1; RMSEC, RMSECV and RMSEP values were one of the least and the RPD value was more than 3.

In my research, baseline correction (baseline), normalize, Savitzky-Golay smoothing (S-G), second derivative (2d), S-G and first derivative $(\mathrm{S}-\mathrm{G}+1 \mathrm{st}), \mathrm{S}-\mathrm{G}$ and second derivative $(\mathrm{S}-\mathrm{G}+$ 2nd) and standard normal variate (SNV) were used to preprocess the raw spectra.
Baseline was mainly used to reduce the effects of background and drift on the spectral signal. S-G was applied to decrease noise, especially in combination and overtones of NIR. The derivation can improve the sensitivity and resolution. SNV was applied to reduce the influence of multiplicative interference of scatter by standardizing the absorbance at each wavelength in the spectrum. ${ }^{25}$ Meanwhile, the numbers of latent factors were investigated coupled with the selection of appropriate pretreatment method. Figure 3 and Table 1 showed the relationship with latent factors and PRESS in different pretreatment methods using leave-one cross validation. The results showed that baseline was the best pretreatment method, in which $R_{\text {cal }}^{2}$ was $0.9951, R_{\text {val }}^{2}$ was 0.9716 and $R_{\text {pre }}^{2}$ was 0.9923 . RMSEC, RMSECV and RMSEP value were 0.51, 1.27 and $1.00 \mathrm{ppm}$, and RPD value was 7.13.

\subsection{Variable selection of $i P L S$ and siPLS}

Chemometric methods of iPLS and siPLS were applied to select variables of Yunkang Oral Liquid. The results of iPLS are shown in Fig. 4. The least RMSEP value of interval number was 12 , in which the wavelength ranged from 1726 to $1871 \mathrm{~nm}$. For siPLS method, the best combinations of interval numbers were 5,11 and 13 , in which the wavelength ranges were 1124.5-1209.5, 1640-1725 and 1811.5$1892.5 \mathrm{~nm}$ (Fig. 5).

\subsection{D-COS assignment of Isopsoralen}

The 2D-COS method was applied to identify the characteristic peaks of Isopsoralen. The synchronous

Table 1. Different pretreatment methods of PLS model.

\begin{tabular}{|c|c|c|c|c|c|c|c|c|}
\hline \multirow[b]{2}{*}{ Pretreatment methods } & \multirow[b]{2}{*}{ Latent factors } & \multicolumn{2}{|c|}{ Calibration set } & \multicolumn{2}{|c|}{ Validation set } & \multicolumn{2}{|c|}{ Prediction set } & \multirow[b]{2}{*}{$\mathrm{RPD}$} \\
\hline & & RMSEC & $R^{2}$ & RMSECV & $R^{2}$ & RMSEP & $R^{2}$ & \\
\hline Raw & 2 & 0.48 & 0.9955 & 1.18 & 0.9745 & 1.05 & 0.9758 & 6.79 \\
\hline Baseline & 3 & 0.51 & 0.9951 & 1.27 & 0.9716 & 1.00 & 0.9923 & 7.13 \\
\hline Normalize & 3 & 0.63 & 0.9926 & 1.27 & 0.9716 & 1.10 & 0.9715 & 6.48 \\
\hline $2 \mathrm{~d}$ & 3 & 1.12 & 0.9736 & 3.37 & 0.7756 & 4.07 & 0.6813 & 1.72 \\
\hline $\mathrm{SG}$ & 2 & 0.68 & 0.9914 & 1.25 & 0.9728 & 0.96 & 0.9776 & 7.43 \\
\hline SG 1st & 4 & 0.23 & 0.9988 & 4.77 & 0.5401 & 4.71 & 0.6042 & 1.51 \\
\hline SG 2nd & 6 & 1.36 & 0.9647 & 6.73 & 0.1867 & 6.11 & 0.0949 & 1.17 \\
\hline SNV & 2 & 0.65 & 0.9921 & 1.29 & 0.9705 & 1.12 & 0.9708 & 6.37 \\
\hline
\end{tabular}

Note: The unit of RMSEC, RMSECV and RMSEP is ppm. 


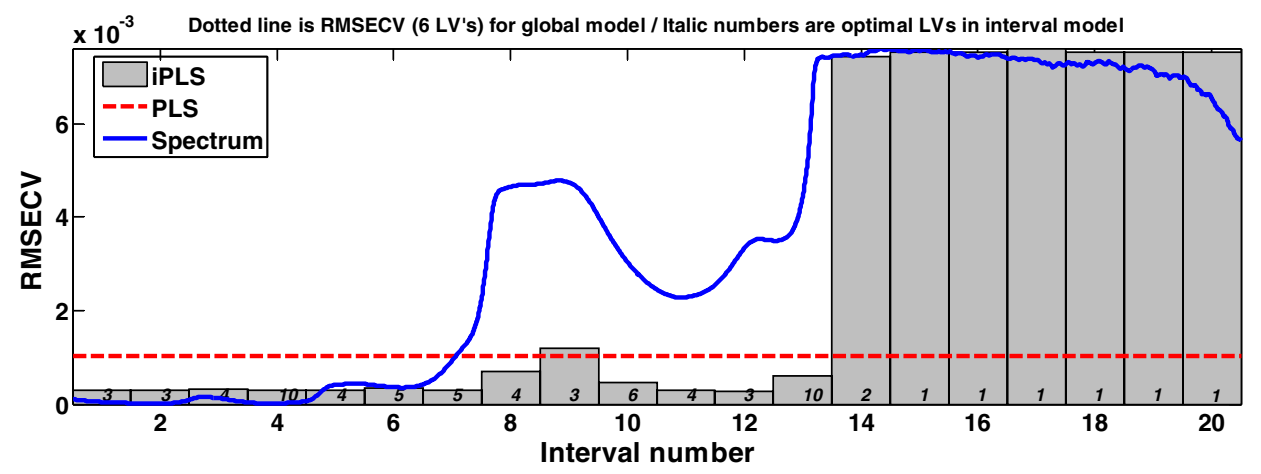

Fig. 4. RMSECV for 20 interval models (bars) and full-spectrum model (black dotted line).

spectrum of deuterated chloroform is shown in Fig. 6(a), the strong peaks of deuterated chloroform including 2180-2320 and 2433-2500 nm. Before assigning the characteristic peaks of Isopsoralen, the strong peaks were eliminated. Therefore, the synchronous spectrum of raw spectra was shown in Fig. 6(b).

The NIR of Isopsoralen was analyzed by 2DCOS method, the characteristic bands including

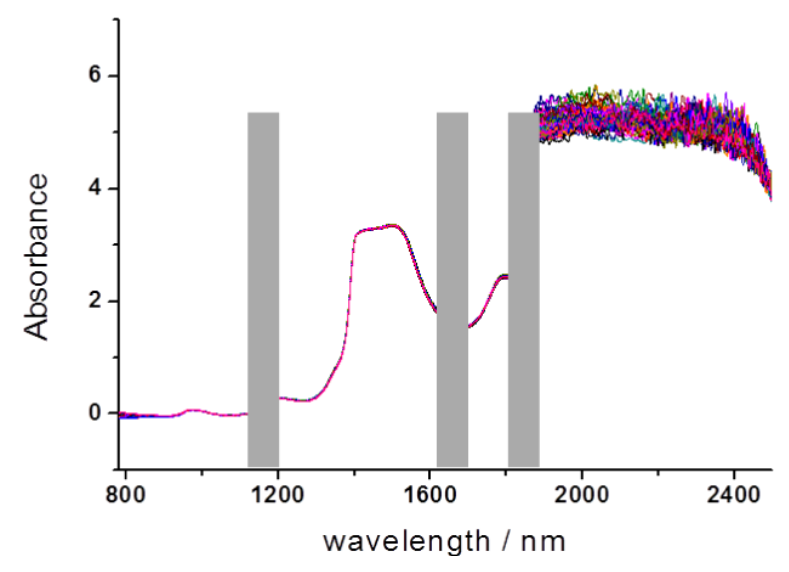

Fig. 5. The results of siPLS.

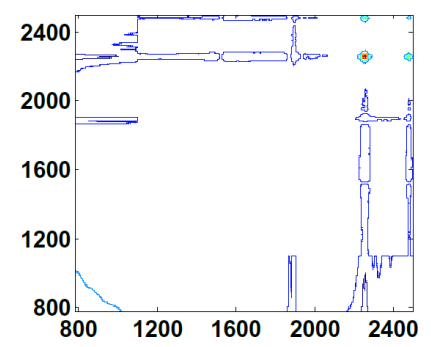

(a)

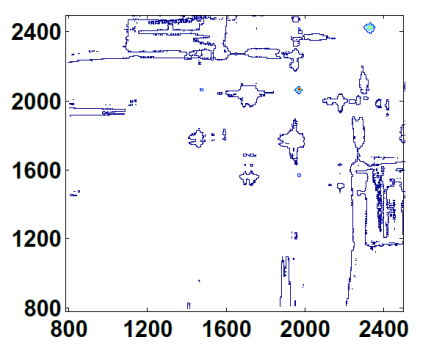

(b)
Fig. 6. The synchronous spectrum of deuterated chloroform (a) and raw spectra of Isopsoralen (b).
1376-1429, 1596-1692 and 2107-2143 nm. According to the structure of Isopsoralen, the band of $1376-1429 \mathrm{~nm}$ is the combination frequency absorption of aromatics C-H, 1596-1692 nm was the absorption of vinyl which connected with oxygen and primary frequency of stretching vibration of $\mathrm{C}-\mathrm{H}$ in aromatic ring, 2107-2143 nm was $\mathrm{C}-\mathrm{H}$ absorption of benzene ring. These bands are assigned to the characteristics of Isopsoralen. ${ }^{26}$ Because of strong absorption of water in combinations and first overtones, 1376-1429 and 1596-1692 nm were chosen to establish the PLS model.

\subsection{The PLS model of Isopsoralen in Yunkang Oral Liquid}

The bands of Chemometrics and 2D-COS were chosen to establish PLS model, respectively. Meanwhile, the numbers of latent factors were investigated coupled with the selection of appropriate bands. Figure 7 and Table 2 showed the relationship with latent factors and PRESS in different variable selection methods using leave-one cross validation.

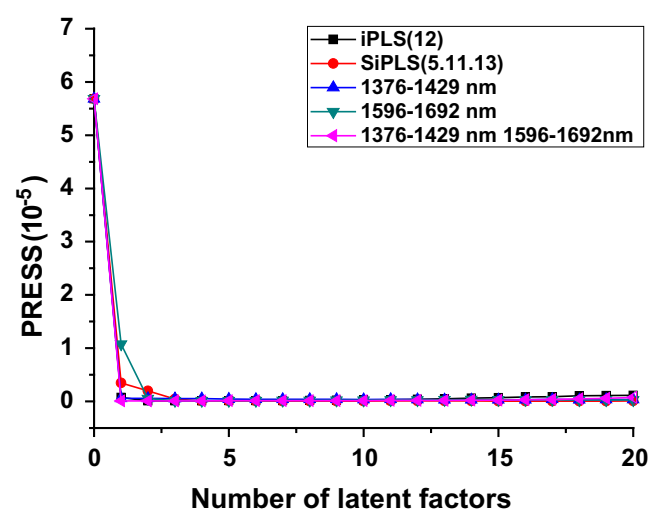

Fig. 7. PRESS plot of NIR by different bands. 
Table 2. The evaluation parameters of PLS model.

\begin{tabular}{|c|c|c|c|c|c|c|c|c|}
\hline \multirow[b]{2}{*}{ Variables selection } & \multirow[b]{2}{*}{ Latent factors } & \multicolumn{2}{|c|}{ Calibration set } & \multicolumn{2}{|c|}{ Validation set } & \multicolumn{2}{|c|}{ Prediction set } & \multirow[b]{2}{*}{ RPD } \\
\hline & & RMSEC & $R^{2}$ & RMSECV & $R^{2}$ & RMSEP & $R^{2}$ & \\
\hline iPLS (12) & 2 & 0.27 & 0.9987 & 0.30 & 0.9985 & 0.23 & 0.9987 & 31.00 \\
\hline $\operatorname{siPLS}(5,11,13)$ & 3 & 0.40 & 0.9970 & 0.67 & 0.9921 & 0.43 & 0.9955 & 16.58 \\
\hline $1376-1429 \mathrm{~nm}$ & 1 & 0.70 & 0.9908 & 0.75 & 0.9902 & 0.66 & 0.9894 & 10.80 \\
\hline $1596-1692 \mathrm{~nm}$ & 2 & 0.64 & 0.9923 & 0.73 & 0.9906 & 0.54 & 0.9931 & 13.21 \\
\hline $1376-1429 \mathrm{~nm} 1596-1692 \mathrm{~nm}$ & 1 & 0.31 & 0.9982 & 0.32 & 0.9982 & 0.22 & 0.9988 & 32.41 \\
\hline
\end{tabular}

Note: 1376 -1429 and 1596-1692 nm were the spectral assignment of Isopsoralen The unit of RMSEC, RMSECV and RMSEP is ppm.

The $R_{\text {cal }}^{2}, R_{\text {val }}^{2}$ and $R_{\text {pre }}^{2}$ of siPLS were $0.9970,0.9921$ and 0.9955, RMSEC, RMSECV, RMSEP and RPD of siPLS were $0.40 \mathrm{ppm}, 0.67 \mathrm{ppm}, 0.43 \mathrm{ppm}$ and $16.58, R_{\text {cal }}^{2}, R_{\text {val }}^{2}$ and $R_{\text {pre }}^{2}$ of iPLS were 0.9987 , 0.9985 and 0.9987, RMSEC, RMSECV, RMSEP and RPD of iPLS were $0.27 \mathrm{ppm}, 0.30 \mathrm{ppm}$, $0.23 \mathrm{ppm}$ and 31.00 . The results were good.

The $R_{\text {cal }}^{2}, R_{\text {val }}^{2}$ and $R_{\text {pre }}^{2}$ of $2 \mathrm{D}-\mathrm{COS}$ spectral assignment were 0.9982, 0.9982 and 0.9988, RMSEC, RMSECV, RMSEP and RPD of 2D-COS spectral assignment were $0.31 \mathrm{ppm}, 0.32 \mathrm{ppm}, 0.22 \mathrm{ppm}$ and 32.41. The result is good as siPLS and iPLS. However, the bands of 2D-COS spectral assignment are more significant and the PLS model was stable and robust. The phenomena in which different bands were chosen by different chemometric methods for the same substance was overcome. It provided a reference method and guidance for the variable selection of NIR application.

\section{Conclusion}

In this study, NIR assignment of Isopsoralen was selected using deuterated chloroform solvent and 2D-COS technology. According to the auto-peaks of 2D-COS synchronous spectrum, the characteristic bands of Isopsoralen included 1376-1429, 15961692 and $2107-2143 \mathrm{~nm}$. Combined with the structure of Isopsoralen, the band $1376-1429 \mathrm{~nm}$ is the combination frequency absorption of aromatics C-H. Band 1596-1692 nm was the absorption of vinyl which connected with oxygen and primary frequency of stretching vibration of $\mathrm{C}-\mathrm{H}$ in aromatic ring. Band $2107-2143 \mathrm{~nm}$ was the $\mathrm{C}-\mathrm{H}$ absorption of benzene ring. NIR collection of Isopsoralen was used as the CQA in different concentrations of Yunkang Oral Liquid, the PLS model using 2D-COS bands, iPLS and siPLS bands obtained good results. Therefore, it provides a reference for NIR spectral assignment of chemical compositions in CMM, and provides guidance for the stable and robust variable selection of NIR application.

\section{Acknowledgments}

This work was financially supported by the National Natural Science Foundation of China (81303218), Doctoral Fund of Ministry of Education of China (20130013120006), and Independent project topics of Beijing University of Chinese Medicine (2014-JYBZZ-XS-082).

\section{References}

1. Y. Ozaki, S. Šašič, J. Jiang, "How can we unravel complicated near infrared spectra? - Recent progress in spectral analysis methods for resolution enhancement and band assignments in the near infrared region," J. Near Infrared Spectrosc. 9, 63-69 (2001).

2. Y. Ozaki, "Near-infrared spectroscopy-its versatility in analytical chemistry," Anal. Sci. 28, 545-563 (2012).

3. D. Cozzolino, H. E. Smyth, M. Gishen, "Feasibility study on the use of visible and near-infrared spectroscopy together with chemometrics to discriminate between commercial white wines of different varietal origins," J. Agric. Food Chem. 51, 77037708 (2003).

4. K. Mitsui, S. Tsuchikawa, "Application of near infrared spectroscopy (NIR) to light-irradiated wood," Eur. J. Wood Wood Prod. 61, 159-160 (2003).

5. I. Noda, "Two-dimensional infrared spectroscopy," J. Am. Chem. Soc. 111, 8116-8118 (1989). 
6. I. Noda, A. E. Dowrey, C. Marcott, G. M. Story, "Generalize two-dimensional correlation spectroscopy," Focal Point. 54, 236-248 (2000).

7. Y. Ozaki, Y. Liu, I. Noda, "Two-dimensional infrared and near-infrared correlation spectroscopy: Applications to studies of temperature-dependent spectral variations of self-associated molecules," Appl. Spectrosc. 51, 526-535 (1997).

8. I. Noda, "Two-dimensional correlation analysis useful for spectroscopy, chromatography, and other analytical measurement," Anal. Sci. 23, 139-146 (2007).

9. H. Liu, J. P. Xu, L. B. Qu, B. R. Xiang, "Generalized two-dimensional correlation near-infrared spectroscopy and principal component analysis of the structures of methanol and ethanol," Sci. China Chem. 53, 1155-1160 (2010).

10. T. Genkawa, M. Watari, T. Nishii, M. Suzuki, Y. Ozaki, "Two-Dimensional heterospectral correlation analysis of water and liquid oleic acid using an online near-infrared/mid-infrared dual-region spectrometer," Appl. Spectrosc. 67, 724-730 (2013).

11. H. Shinzawa, K. Awa, I. Noda, Y. Ozaki, "Twodimensional (2D) band shift correlation spectroscopy for near-infrared (NIR) imaging data," J. Mol. Struct. 974, 25-29 (2010).

12. S. Šašić, M. Morimoto, M. Otsuka, Y. Ozakia, "Two-dimensional correlation spectroscopy as a tool for analyzing vibrational images," Vib. Spectrosc. 37, 217-224 (2005).

13. Z. S. Wu, C. L. Sui, B. Xu, L. Ai, Q. Ma, Y. J. Qiao, "Multivariate detection limits of on-line NIR model for extraction process of chlorogenic acid from Lonicera japonica," J. Pharm. Biomed. 77, 16-20 (2013).

14. X. F. Wang, Y. F. Bao, G. L. Liu, G. Li, L. Lin, "Study on the best analysis spectral section of NIR to detect alcohol concentration based on SiPLS," Procedia Eng. 29, 2285-2290 (2012).

15. H. Jiang, G. H. Liu, C. L. Mei, S. Yu, X. H. Xiao, Y. H. Ding, "Measurement of process variables in solid-state fermentation of wheat straw using FTNIR spectroscopy and synergy interval PLS algorithm," Spectrochim Acta A 97, 277-283 (2012).

16. Q. S. Chen, J. W. Zhao, M. H. Liu, J. R. Cai, J. H. Liu, "Determination of total polyphenols content in green tea using FT-NIR spectroscopy and different PLS algorithms," J. Pharm. Biomed. 46, 568-573 (2008).

17. Q. Yang, S. Zhou, Q. H. Zhu, J. D. Yang, "Simultaneous determination of magnolol and hokiol in HuoxiangZhengqi oral liquid using nearinfrared spectrometry," J. Southwest China Normal Univ. 36, 38-42 (2011).

18. Y. Bai, Y. L. Guo, J. W. Lei, "Rapid determination of Magnolol and Honokiol in Xiangshayangwei pill by near-infrared spectroscopy," Chin. J. Modern Appl. Pharm. 29, 1022-1025 (2012).

19. Chinese Pharmacopoeia Commission, Pharmacopeia of People's Republic of China, China Medical Science Press, Beijing (2010).

20. C. Q. Ke, "Determination of baicaline in yunkang oral liquid by HPLC," Anhui Med. Pharm. J. 10, 428-429 (2006).

21. Y. L. Pei, Y. F. Peng, X. N. Pan, Z. S. Wu, Y. J. Qiao, "NIR assignment of Magnolol by 2D-COS Technology," Beijing, Science paper Online, Available at http://www. paper.edu.cn/html/releasepaper $/ 2014 / 01 / 964 /$.

22. C. J. Wu, J. H. Ge, H. R. Tang, "Determination the contents of psoralen and isopsoralen in yunkang oral liquid by HPLC ", China Pharmacist. 1, 429-430 (2008).

23. R. Buchet, Y. Wu, G. Lachenal, Y. Ozaki, "Selecting two-dimensional cross-correlation functions to enhance interpretation of near-infrared spectra of proteins," Appl. Spectrosc. 55, 155-162 (2001).

24. L. Thierry, P. Michel, "Importance of the reference spectrum on generalized two-dimensional correlation spectroscopy: Relation between intensity variations and synchronism," J. Phys. Chem. A 107, 6366-6372 (2003).

25. X. L. Chu, Molecular Spectroscopy Analytical Technology Combined with Chemometrics and Its Application, Chemical Industry Press, Beijing (2010).

26. W. Jerry, J. L. Weyer, Practical Guide and Spectral Atlas for Interpretive Near-Infrared Spectroscopy, 2nd Edition, CRC Press, Boca Raton (2012). 